\title{
Repeat Testing of Antibodies and Complements in Systemic Lupus Erythematosus: When Is It Enough?
}

\author{
Thomas C. Raissi, Carly Hewson, and Janet E. Pope
}

ABSTRACT. Objective. Patients with systemic lupus erythematosus (SLE) frequently undergo repeat testing for antibodies against extractable nuclear antigens (anti-ENA), but it is not known whether this is necessary or cost-effective. This study characterized the frequencies of changes in anti-ENA, anti-dsDNA, and complement C3 and C4 upon repeat testing.

Methods. Chart review was done at one site of 130 patients with SLE enrolled in the 1000 Canadian Faces of Lupus prospective registry with annual antibody and complement testing. We determined the frequency of seroconversion (changes) on the next test and over the entire followup given 1 or multiple consistent results, and the cost to detect these changes.

Results. Overall, $89.4 \%$ of patients had no changes in anti-ENA screening results from the first available test, $3.3 \%$ changed from negative to positive, and $7.3 \%$ from positive to negative. Following a single anti-ENA test, $3.9 \%$ of negative tests changed to positive and $4.2 \%$ of positive changed to negative on the next test. After multiple consistent tests, the frequencies of changes progressively declined. No changes from the first test were observed in anti-dsDNA, C3, and C4 in $60.8 \%, 83.3 \%$, and $75.4 \%$ of patients, respectively. After 2 consistent anti-ENA tests, the cost to detect 1 change was above US\$2000.

Conclusion. Anti-ENA results change infrequently, especially following 1 or more negative tests. The high cost and lack of evidence that changes affect management suggest that repeating anti-ENA tests routinely is unnecessary. Anti-dsDNA and complements change more frequently after an abnormal result, but less after a normal value. (First Release April 15 2018; J Rheumatol 2018;45:827-34; doi:10.3899/jrheum.161365)

Key Indexing Terms: SYSTEMIC LUPUS ERYTHEMATOSUS COMPLEMENTS ANTI-dSDNA

Systemic lupus erythematosus (SLE) is an autoimmune connective tissue disease that may affect virtually any organ in the body. SLE autoimmunity is associated with antinuclear antibodies (ANA) ${ }^{1}$. These react with antigens including dsDNA, histone proteins, centromere proteins, and extractable nuclear antigens (ENA). ENA commonly include Sm antigen, La/SSB, RNP, and Scl-70, and often include Ro/SSA.

From the University of Western Ontario, Schulich School of Medicine and Dentistry, Department of Medicine; Division of Rheumatology,

Department of Medicine, St. Joseph's Health Care, London, Ontario, Canada.

A part of this work was supported by the Program of Experimental Medicine (POEM), Department of Medicine, University of Western Ontario.

T.C. Raissi, BSc, University of Western Ontario, Schulich School of Medicine and Dentistry; C. Hewson, MD, FRCPC, BSc, University of Western Ontario, Schulich School of Medicine and Dentistry, Department of Medicine; J.E. Pope, MD, MPH, FRCPC, University of Western Ontario, Schulich School of Medicine and Dentistry, Department of Medicine, and Professor of Medicine, Division Head, Division of Rheumatology, St. Joseph's Health Care.

Dr. T.C. Raissi and Dr. C. Hewson contributed equally to this work and are co-first authors.

Address correspondence to Dr. J.E. Pope, 268 Grosvenor St., London, Ontario N6A4V2, Canada.E-mail: janet.pope@sjhc.london.on.ca Accepted for publication December 14, 2017.

\section{EXTRACTABLE NUCLEAR ANTIGENS IMMUNOLOGIC TESTING COSTS}

ANA, used for screening if SLE is suspected, are 95\% sensitive and often the first abnormal autoimmune result, but they lack specificity ${ }^{2,3}$. Once found to be positive, there is no value in serial measurements ${ }^{4}$. Anti-ENA and anti-dsDNA are ordered to detect antibodies against specific nuclear components if the ANA test is positive. Antibodies to dsDNA, present in 40-60\% of patients with SLE, are associated with renal involvement ${ }^{3,5}$. Anti-dsDNA levels correlated with or predicted flares in longitudinal studies $^{5,6,7,8}$. Anti-Sm antibodies are specific for SLE, and are seen in $30-40 \%$ of patients ${ }^{3,9}$. Anti-Ro/SSA and anti-La/SSB are associated with photosensitivity, subacute cutaneous lupus erythematosus, neonatal SLE, and Sjögren disease $^{3}$.

SLE involves immune complex formation. This consumes complement components (C3, C4, CH50), which are routinely measured in SLE. C3 and/or C4 are reduced and $\mathrm{CH} 50$ is increased during flares, and complement is used in addition to anti-dsDNA to monitor disease activity, particularly renal involvement ${ }^{1,10,11,12}$.

SLE is diagnosed by physician clinical judgment, and classification criteria may be used [1997 American College of Rheumatology (ACR) or 2012 Systemic Lupus International Collaborating Clinics (SLICC) criteria] ${ }^{13,14,15}$.

Personal non-commercial use only. The Journal of Rheumatology Copyright (c) 2018. All rights reserved. 
Both include ANA, anti-dsDNA, and anti-Sm; SLICC criteria also include low complement ${ }^{13,14,15}$. Disease activity may be followed using the SLE Disease Activity Index, which includes anti-dsDNA and complement ${ }^{16}$.

ANA and anti-ENA are commonly serially repeated after diagnosis ${ }^{17,18}$. Evidence suggests that repeat ANA measurements are not indicated in ANA-positive SLE; they neither change significantly nor are helpful in monitoring disease activity ${ }^{19}$. The utility of repeating anti-ENA is not established. Widely varying frequencies of anti-ENA changes in serially tested patients with SLE have been documented ${ }^{20,21,22}$. Anti-dsDNA and complement are routinely repeated as markers of disease activity in SLE, but it is not known how frequently these tests should be repeated if normal initially.

Immunologic testing contributes to the costs of SLE. Clinical practice guidelines, including the Choosing Wisely campaign's guidance, advise against repeating ANA and simultaneous ordering of ANA with anti-dsDNA and anti-ENA ${ }^{19,20,23}$. Incremental costs of serially repeating anti-ENA are unknown.

This study investigated the frequency of changes of anti-ENA at 1 center. We theorized, based on clinical experience, that seroconversion of anti-ENA would be uncommon and serial measurements would be unlikely to change from baseline testing; therefore our intention was to study when to stop reordering tests that may lack future utility. We determined the frequency of changes in the next test and over the entire followup after 1 or multiple consistent results of anti-ENA, anti-dsDNA, and complement C3 and $\mathrm{C} 4$, and calculated the cost of detecting each change.

\section{MATERIALS AND METHODS}

Our study included patients enrolled in the 1000 Canadian Faces of Lupus study at St. Joseph's Hospital in London, Ontario, Canada. Ethics approval was obtained from the University of Western Ontario Health Sciences Research Ethics Board (\#11659E) and each subject signed a letter of information. The 1000 Canadian Faces of Lupus is a national prospective observational cohort of patients with SLE, recruiting cases of SLE since 2005. Inclusion criteria for subjects of this study were (1) meeting the ACR criteria for $\mathrm{SLE}^{13}$, and (2) having at least 1 repeat test available for anti-ENA, anti-dsDNA, or complement, at least 1 year after the initial test. From January 1999 through February 2016, 137 patients were reviewed. Data were prospectively collected annually as part of the protocol.

The majority of tests were processed in the London Health Sciences Centre immunology laboratory. However, some results were obtained from private laboratories and other hospital laboratories throughout southwestern Ontario using the Ontario Laboratories Information System.

Our center uses an automated EUROIMMUN ELISA for both anti-dsDNA and anti-ENA. The anti-dsDNA is a quantitative assay. Anti-ENA is semiquantitative. Automated assays have been in use since 2012. Between 2005 and 2012, the EUROIMMUN assays were set up manually. From 1999 to 2005, ELISA assays were completed manually using Varelisa plates from Somagen. C3 and C4 levels between 1999 and the present date were determined quantitatively using nephelometry (Beckman Coulter). We do not have information about the specific tests used from other external laboratories, but have the normal ranges for all tests.

We determined the frequency of change in repeat measurements of anti-ENA antibodies, anti-dsDNA, C3, and C4. Four patterns were possible for each antibody: always negative, negative at initial testing with positive seroconversion at any point thereafter, positive at initial testing with negative seroconversion thereafter, and always positive. C3 and C4 were expressed as always normal [including higher than the upper limit of normal (ULN)], normal at initial testing with change to low, low at initial testing with subsequent change to normal, and always low. Frequencies were calculated for each possible pattern. We expected fluctuation in anti-dsDNA, C3, and C4, so the proportion of patients with 2 or more consecutive initial normal results who had a subsequent abnormal result at any point during followup was calculated.

To estimate the likelihood of change following multiple consistent test results, for each test we identified all possible intervals during which a patient had consistent results. If a test was performed more frequently than once a year, we counted each calendar year as 1 test. We defined high-titer anti-dsDNA as triple the threshold for positive anti-dsDNA specified by the laboratory. Test results reported as "borderline positive" or "equivocal" were classified as consistent with whatever result preceded them.

We estimated the cost of detecting test changes in 2 ways (low and high cost). We used the national limit from the US Medical Laboratory Fee Schedule as the lower limit (codes 86235 for anti-ENA screen and each subserology, 86225 for anti-dsDNA, and 86160 for C3 or C4 $)^{24}$. To estimate the higher costs charged to individuals and private insurance, we also calculated costs to detect each change using prices obtained from a major US commercial laboratory in which each antibody within the ENA is analyzed and has its own cost. We calculated the cost of detecting a change given the number of previous consistent years by dividing the total test cost (for each serial measurement) by the fraction of tests changed. For example, after a single negative anti-ENA screen result, 11 out of the subsequent 280 tests were positive. If the test costs US $\$ 24.43$, the cost to detect a change was $\$ 24.43 \div(11 \div 280)=\$ 621.85$ (this is the same as multiplying the cost per test by the number of tests done and dividing by the number of positive tests).

The low cost estimate included only the cost of the specific test we aimed to detect a change in, based on the assumption that all tests described here are ordered independently. For example, the cost of detecting a change in anti-ENA screening did not include costs of additional subserologies that many laboratories automatically perform following a positive anti-ENA screen. Calculating the cost to detect changes while accounting for bundled tests, or for automatic subserology tests performed following positive anti-ENA screen results, is complicated and the result may be misleading. Our calculations therefore assume that each test is performed in isolation. While we reported the frequency of changes regardless of how many test results were available, we estimated costs only if there were 100 or more test results. The high cost estimate would occur if a commercial laboratory performed all subserologies without an overall screen for ENA. For instance, if someone orders an ENA, it should not be further tested if the overall screen is negative. However, some laboratories perform each serology separately (SSA, SSB, Sm, RNP, and Scl-70). Other laboratories include Jo1 antibody, and various subtypes of RNP (U1RNP) and 2 different anti-Ro tests. To be conservative in our estimates, we concentrated on the 5-panel ENA even for the high cost estimates. Therefore, in the high-cost scenario, 5 antibodies within the ENA would be performed with each ENA screen and would cost far more (adding the cost of each antibody each time an ENA was ordered).

\section{RESULTS}

Of 137 patients enrolled in the study at our site, complete data were available for 131 patients. One patient was excluded because no tests had been repeated, and 130 patients were included. Their characteristics at recruitment to the 1000 Canadian Faces of Lupus study are shown in Table 1. When the anti-ENA panel is ordered, a screen is initially performed, and subserologies are measured only if that screen is positive. The 123 patients with 2 or more anti-ENA results available had a mean 5.4 (SD 1.9) measure-

Personal non-commercial use only. The Journal of Rheumatology Copyright @ 2018. All rights reserved. 
Table 1. Baseline demographic and disease variables in patients with SLE included in this study compared to the entire "The 1000 Faces of Canadian Lupus" cohort. Values are n (\%) unless otherwise specified.

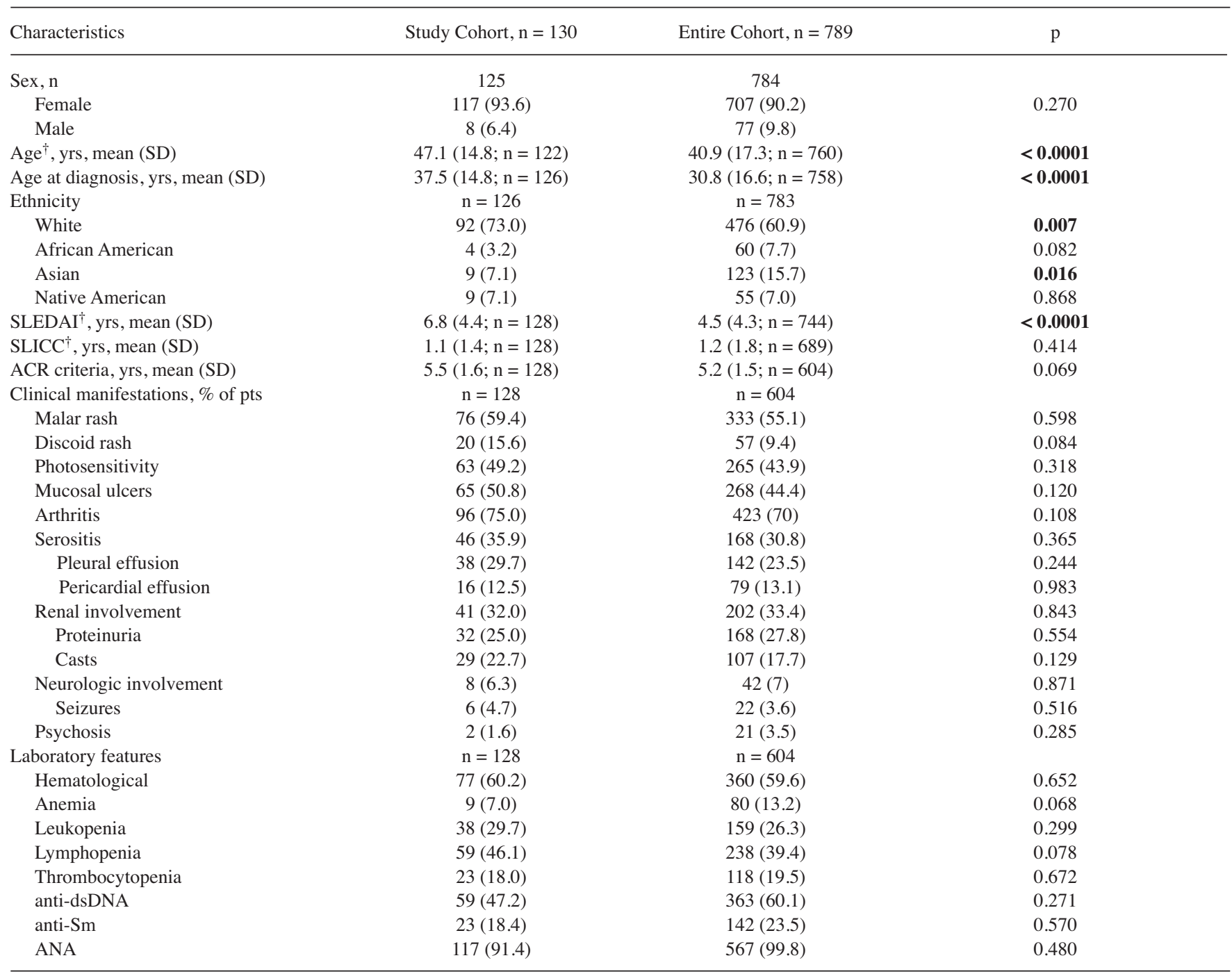

$\dagger$ At enrollment. Characteristics were calculated by measures of central tendency and proportions. Student $t$ test or Mann-Whitney U test was applied for comparison between continuous variables, as well as chi-square test for categorical variables. Significant data are in bold face. SLE: systemic lupus erythematosus; SLEDAI: Systemic Lupus Erythematosus Disease Activity Index; SLICC: Systemic Lupus International Collaborating Clinics; ACR: American College of Rheumatology; ANA: antinuclear antibodies.

ments per patient (Figure 1). Every subsequent anti-ENA screen result was the same as the first in $89.4 \%$. Changes occurred in a minority of patients: $3.3 \%$ of patients were initially negative and seroconverted to positive while $7.3 \%$ of patients were initially positive and seroconverted to negative over the followup period.

The most frequently detected anti-ENA autoantibodies were anti-Ro (initially positive in $37.4 \%$ ) and anti-RNP (initially positive in $15.4 \%$ ). Only $4.9 \%$ of patients had changes (either negative or positive) in anti-Ro/SSA, 2.4\% in anti-La/SSB, 3.3\% in anti-RNP, and 3.3\% in anti-Sm. No patients were positive for anti-Scl-70 at any time.

There were higher rates of changes for anti-dsDNA. For the 130 patients with 2 or more anti-dsDNA results available, a mean of 7.2 (SD 3.1) measurements were available per patient (Figure 1). During followup, 60.8\% of patients experienced no change in anti-dsDNA, $17.7 \%$ were initially negative for anti-dsDNA and converted to positive at some point, and $21.5 \%$ of patients were initially positive and converted to negative.

Complement $\mathrm{C} 3$ and $\mathrm{C} 4$ levels also showed fluctuations in our study group. The majority of patients always had normal complement levels. Of the 126 patients with 2 or more complement tests available, a mean of 7.3 (SD 3.3) measurements were available per patient (Figure 1). Results did not change for $\mathrm{C} 3$ in $83.3 \%$ and for $\mathrm{C} 4$ in $75.4 \%$ of patients over the followup period. For $\mathrm{C} 3,11.1 \%$ of patients initially had normal values which became low and for $\mathrm{C} 4$ this

Personal non-commercial use only. The Journal of Rheumatology Copyright $\odot$ 2018. All rights reserved. 
Number of patients

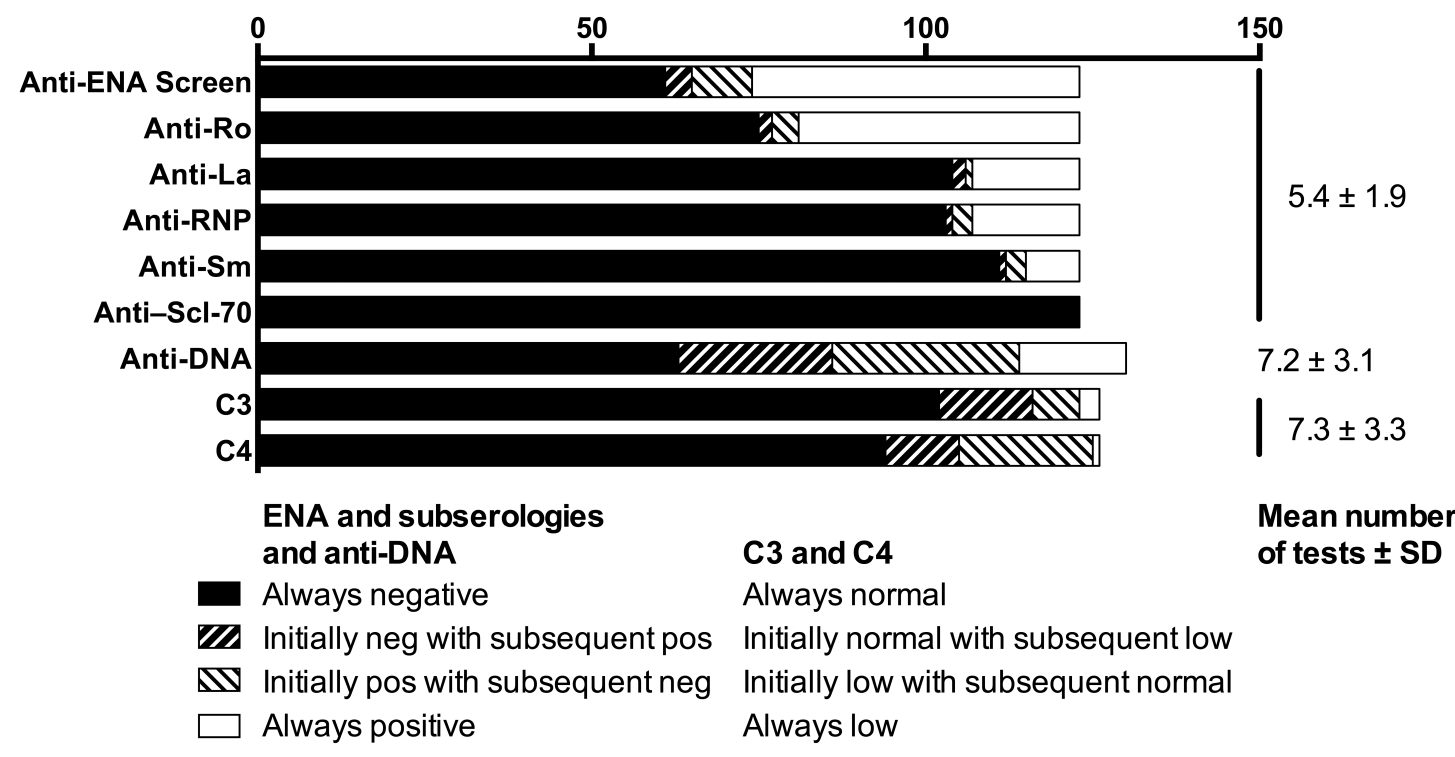

Figure 1. Changes in anti-ENA and subserology, anti-dsDNA, and complement status compared to earliest available test. ENA: extractable nuclear antigens; neg: negative; pos: positive; C3: complement factor 3; C4: complement factor 4.

occurred in $8.7 \%$. For C3, 5.6\% initially had low levels that normalized, and $15.9 \%$ for $\mathrm{C} 4$.

Although there was variability on repeat testing of anti-dsDNA and $\mathrm{C} 3$ and $\mathrm{C} 4$, in individuals with many negative (normal) tests, there was a progressively decreasing probability of a future positive test. In the 70 patients with 3 sequential negative anti-dsDNA tests, $78.6 \%$ remained negative on repeat testing thereafter. In the 99 patients with 3 sequential normal C3 tests, 92.9\% remained normal on further repeat testing. Eighty-seven patients had 3 sequential normal C4 tests, and $96.5 \%$ remained normal thereafter.

An important clinical question is whether to repeat an anti-ENA test given 2 or more previous consistent results, especially if these results were normal. We determined the frequency that the next result would be different after 1 or more previous tests showed consistent results. Following any single anti-ENA screen, $3.9 \%$ of negative anti-ENA tests changed to positive ( 280 tests) and $4.2 \%$ of positive changed to negative (263 tests) on the next test (Figure 2). These frequencies became progressively lower after more years of consecutive consistent tests. No changes in anti-ENA or any subserology were observed after 5 or more consecutive consistent tests.

Quantifying the frequency of anti-dsDNA changes after multiple consistent years showed that while 7.9\% (573 tests) and $24.5 \%$ (273 tests) of negative and positive anti-dsDNA results changed respectively after 1 test; after 2 through 7 consistent years these frequencies never exceeded $4 \%$ and $16 \%$, respectively (Figure 3 ). We investigated whether anti-dsDNA would revert to normal less frequently after multiple consistent tests with an anti-dsDNA titer 3-times the ULN or higher. Unexpectedly, positive anti-dsDNA results following 1 or more consistent years with a high titer occurred less frequently than following any positive anti-dsDNA result. While a majority of tests following a single year of low complement tests reverted the following year, there was a progressively lower frequency of tests reverting to normal following multiple low results.

We estimated, for each serology, the cost to detect 1 change following multiple normal results (Table 2). For the cost of each test, we used the Medicare National Limit from 2016. The Medicare costs of each test were $\$ 24.43$ for anti-ENA screen, $\$ 18.71$ for anti-dsDNA, and $\$ 16.35$ for C3 or $\mathrm{C}^{24}$. To estimate the higher costs charged to individuals and private insurance, we calculated costs to detect each change using prices $\$ 124, \$ 124$, and $\$ 83$, respectively. Table 2 contains estimates for the costs of detecting changes to abnormal results using Medicare costs as well as the commercial laboratory's fees; but for clarity, only Medicare costs are reported in the text below.

At some institutions, the anti-ENA panel is ordered as 1 combined panel, while at others each subserology is ordered and performed separately. If an anti-ENA screen is automatically followed by 5 subserologies, this could potentially increase the cost if a laboratory charges for each test.

After a single negative anti-ENA screen, the expense required to detect 1 anti-ENA change to positive the next year was $\$ 621.85$. After 2 or more consistent years, the costs to Medicare exceeded $\$ 2000$ and $\$ 3000$, respectively. These costs do not include those necessary to determine which anti-ENA subserology was positive, because that will vary according to the laboratory testing algorithm and whether costs are separately charged for each antibody.

Detecting 1 anti-dsDNA change from negative to positive Personal non-commercial use only. The Journal of Rheumatology Copyright $\odot$ 2018. All rights reserved. 

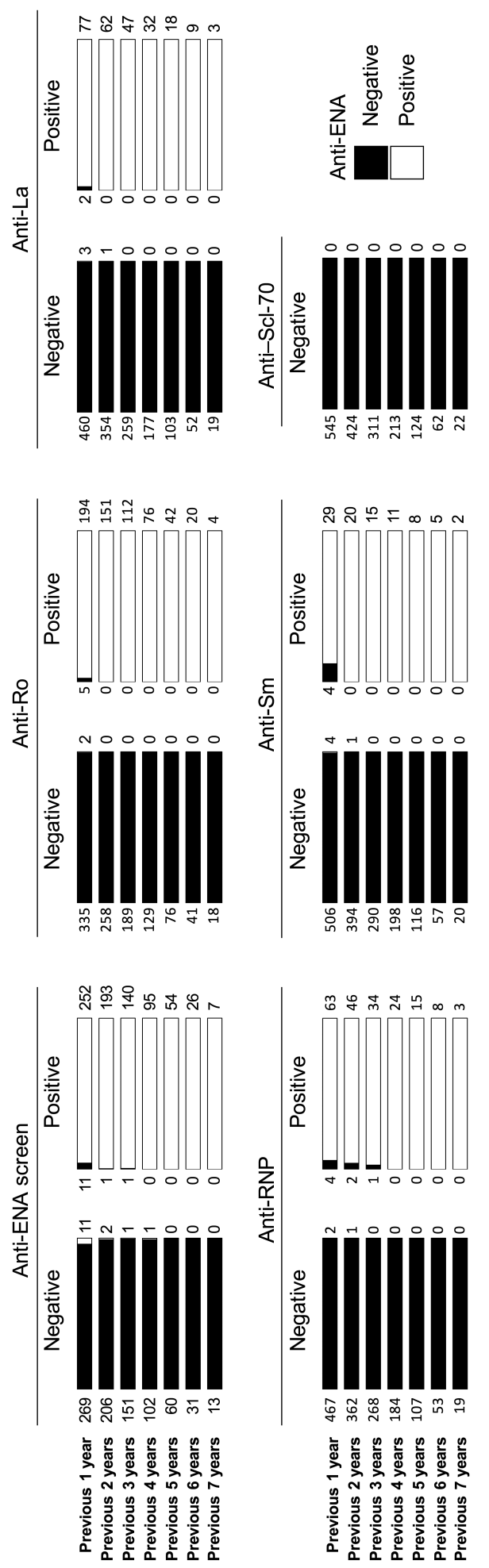

Figure 2. Changes in anti-ENA and subserology status after multiple consistent tests. For each patient, all possible intervals during which each test result was consistent were tabulated. The result of the next test performed is reported according to the number of years in that consistent interval in which the test was performed. ENA: extractable nuclear antigens. cost \$238.24 after 1 anti-dsDNA test. Because of the more consistent frequency of anti-dsDNA changes, the cost of detecting 1 negative-to-positive anti-dsDNA change remained below $\$ 700$, even after 4 consistent years. The costs of detecting low complement $\mathrm{C} 3$ or $\mathrm{C} 4$ following 1 normal result were $\$ 218.79$ and $\$ 216.12$, respectively. Costs to Medicare increased above $\$ 400$ following 2 or more consistent years, but even after 4 consecutive years did not exceed $\$ 1300$ (Table 2).

\section{DISCUSSION}

Frequent serologic monitoring in SLE contributes to healthcare costs. Consistent with our clinical observations, we documented a low frequency of changes $(<5 \%$ over the study) among anti-ENA autoantibodies in patients with SLE enrolled in a prospective cohort. These data suggest that it is not necessary to repeat anti-ENA testing during routine followup, because the initial test result is unlikely to change. There were significant costs associated with detecting infrequent changes. Although we did not investigate the clinical effect of anti-ENA changes, previous authors have demonstrated the absence of correlation between anti-ENA autoantibody concentrations and changes in disease activity ${ }^{25}$.

Exceptions could include pregnancy, where repeat screening for anti-Ro/SSA may be indicated because a positive test would prompt fetal cardiac ultrasound screening to search for congenital heart block, and treatment of the mother with dexamethasone if heart block occurs. Pregnancy in a woman with SLE carries a risk for fetal mortality and morbidity ${ }^{26}$. Repeat anti-ENA testing may also be useful when the diagnosis is in question, because a small number of patients with SLE are ANA-negative but Ro/SSA-positive ${ }^{27}$. We did not study those patients.

Based on clinical experience, we hypothesized that changes in anti-ENA antibodies would be infrequent, which was supported by our data. However, other authors have described higher rates of seroconversion among anti-ENA autoantibodies ${ }^{20,21,22}$. The largest study of 130 patients showed rates of seroconversion of over $50 \%$ for some anti-ENA autoantibodies over 10 years ${ }^{20}$. These high rates of fluctuation were reported specifically in patients positive (at any time over the followup period) for a given autoantibody. Interestingly, our data reproduced their finding of more frequent changes of positive anti-ENA to negative than vice versa, even though changes were lower in both categories in our patients. Differences in results could be related to different laboratory techniques for anti-ENA measurement, or different populations. Most with negative anti-ENA panels remained negative over 10 years in 61 patients with SLE ${ }^{21}$. There was some fluctuation among those patients positive for anti-Ro/SSA and anti-La/SSB, but absolute numbers were similar to those reported here. Praprotnik, et al studied 16 patients with SLE and 15 patients with Sjögren syndrome,

Personal non-commercial use only. The Journal of Rheumatology Copyright @ 2018 . All rights reserved. 


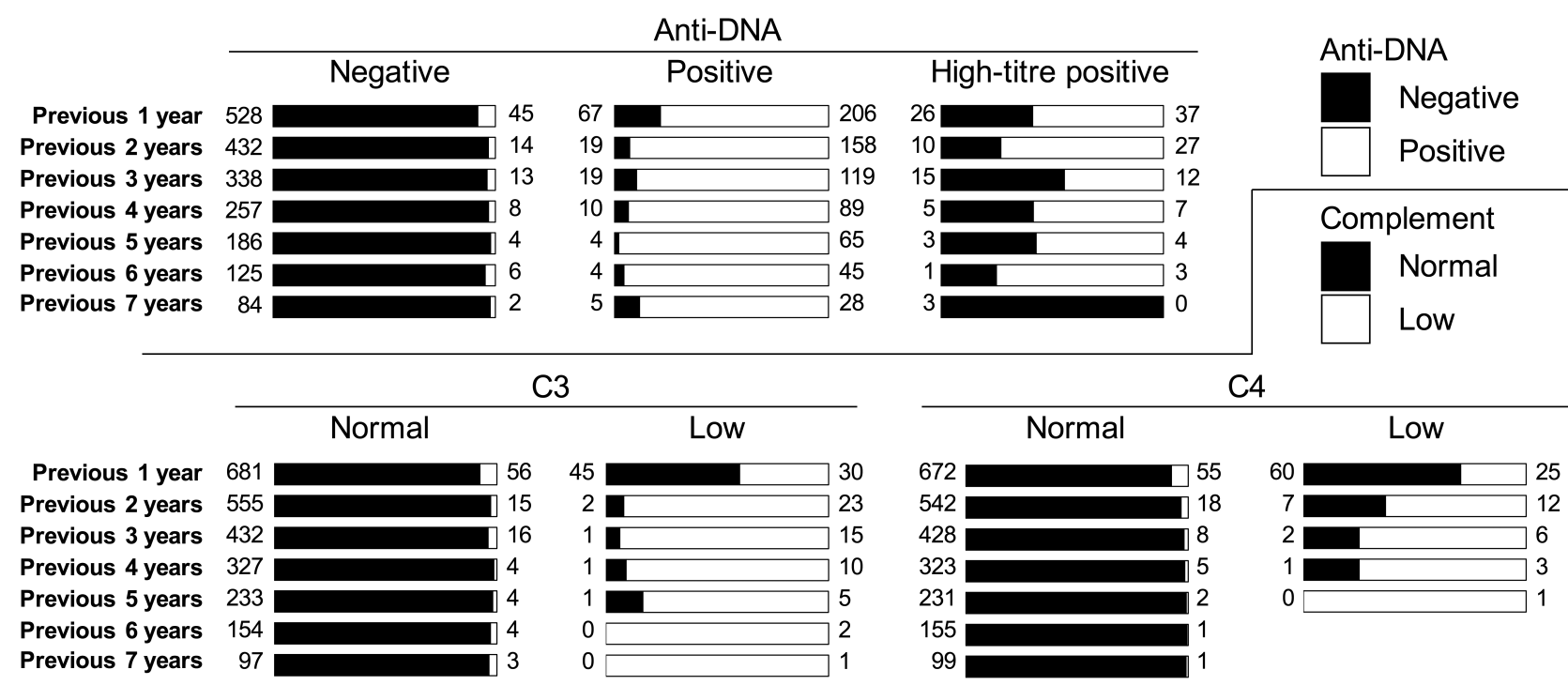

Figure 3. Changes in anti-dsDNA and complement status after multiple consistent tests. For each patient, all possible intervals during which each test result was consistent were tabulated. The result of the next test performed is reported according to the number of years in that consistent interval in which the test was performed. C3: complement factor 3; C4: complement factor 4.

Table 2. Cost of detecting a change in serologic tests after previous normal results. Costs in US dollars. Total numbers of tests used to calculate these estimates are shown in Figure 2.

\begin{tabular}{lcccc}
\hline $\begin{array}{l}\text { Variables } \\
\text { Negative test at a point in time }\end{array}$ & 1 & \multicolumn{3}{c}{ Yrs after a Normal Test } \\
\hline Medicare costs & & 2 & 3 & 4 \\
$\quad$ Anti-ENA neg to pos & 621.85 & 2540.72 & 3713.36 & 2516.29 \\
Anti-DNA neg to pos & 238.24 & 596.05 & 505.17 & 619.77 \\
C3 normal to low & 218.79 & 577.70 & 415.90 & 1213.99 \\
C4 normal to low & 216.12 & 503.22 & 880.86 & 1062.75 \\
Commercial laboratory costs & & & & \\
Anti-ENA neg to pos & 3156.36 & 12,896 & 18,848 & 12,772 \\
Anti-DNA neg to pos & 1578.93 & 3950.29 & 3348.00 & 4107.50 \\
C3 normal to low & 1110.69 & 2932.67 & 2111.31 & 6162.75 \\
C4 normal to low & 1097.11 & 2554.56 & 4471.62 & 5395.00 \\
\hline
\end{tabular}

ENA: extractable nuclear antigens; neg: negative; pos: positive; C3: complement factor 3; C4: complement factor 4.

all anti-Ro/SSA-positive with some changes in titers, but all stayed anti-Ro/SSA-positive ${ }^{22}$.

Unlike the anti-ENA results, patients with abnormal anti-dsDNA and complement results frequently reverted to normal, and this persisted after many consistent years, especially for anti-dsDNA. Interestingly, following an anti-dsDNA test with a titer 3 or more times the ULN had a higher chance of changing to negative - at least $25 \%$ even after 7 consistent years. We hypothesize that this may reflect escalation of therapy or that some patients with high anti-dsDNA could have more fluctuations, although we did not investigate whether test results correlated with any relevant change in clinical SLE disease activity. Two reasons could explain why anti-ENA is stable, but anti-dsDNA varies. First, the underlying pathophysiologic changes occurring in
SLE may have a greater effect on anti-dsDNA than other autoantibodies over time. Second, anti-dsDNA is usually titered or given a numerical value whereas anti-ENA components are often just reported as positive or negative. Changes in anti-ENA from positive to negative were very infrequent; perhaps future treatments may be able to normalize anti-ENA subserologies, which is uncommon with current immunosuppressives.

We calculated costs of detecting 1 change in anti-ENA screening in excess of $\$ 2000$ after 2 consistent normal tests as a low cost estimate using Medicare reimbursement. Private laboratory fees for the tests we studied were about 5-times Medicare reimbursement rates. Many laboratories, including ours, perform this anti-ENA screen that can identify when any subserology is positive, but not provide which sub- 
serology is positive; the cost is the same as for 1 subserology alone. We reasoned that using this screen might increase the cost-effectiveness of detecting positive anti-ENA screens in patients with previously negative anti-ENA screens. After a single negative anti-ENA screen result, subsequently, 11 out of 280 (3.9\%) tests were positive, so $96 \%$ of the tests are uninformative (could be considered a waste of money because there is no value in serially negative tests). The costs are even greater if they are performed more than twice because the likelihood of seroconversion becomes even lower. At centers that do not perform an anti-ENA screen, but immediately perform all subserologies, costs to detect changes are higher.

An important potential cause of variations in seroconversion rates between institutions and points in time is laboratory methodology. The anti-ENA and anti-dsDNA results reported here are derived from manual, and later in the series, automated ELISA. Our study was not designed to compare seroconversion rates across these methods. This is a limitation of real-world data. However, most of the ELISA were performed at the same university hospital laboratory for ENA and anti-dsDNA. There were no obvious predictors of those who seroconverted regarding ENA and those who did not, but we do not know whether positive ENA that became negative were more borderline positives and vice versa because antibodies in general were not titered. This study includes data derived from automated ELISA, which may have improved reliability and reduced random fluctuations compared to previous reports using older techniques ${ }^{20,21,22}$.

Our study has limitations. The data were from a single site. They included both incident and prevalent cases; it is plausible that test results would be most likely to change soon after SLE diagnosis. We did not assess whether changes in test results correlated with any clinical or treatment event, which precludes us from estimating the clinical utility of a changed test. The cost data must be interpreted by clinicians regarding the value that a change in a test could provide. We did not study anticardiolipin antibodies, which can be used to fulfill 1 criterion in the ACR and SLICC classification criteria, but require repeating ( 2 positives $)^{13,14,15}$. Most but not all the tests were performed at a single laboratory, a factor that may have reduced variability, but may limit applicability to patients whose tests are performed in multiple laboratories.

Overall, we found that SLE patients with normal immunologic tests experienced infrequent changes over time and that the cost associated with detecting these changes was high. Following a normal result, the frequency of anti-dsDNA and complement changes was somewhat higher than the very low frequency of anti-ENA changes. While positive (abnormal) anti-ENA results also rarely changed, abnormal anti-dsDNA and complement results frequently reverted to normal, even after multiple consistent abnormal years. While we did not investigate the clinical implications of changes, published data show little predictive value of anti-ENA. Routine annual
anti-ENA testing is likely not necessary in SLE patients with consistent results, especially if they are negative.

\section{REFERENCES}

1. Tsokos GC. Systemic lupus erythematosus. N Engl J Med 2011;365:2110-21.

2. Arbuckle MR, McClain MT, Rubertone MV, Scofield RH, Dennis GJ, James JA, et al. Development of autoantibodies before the clinical onset of systemic lupus erythematosus. N Engl J Med 2003;349:1526-33.

3. Lam GK, Petri M. Assessment of systemic lupus erythematosus. Clin Exp Rheumatol 2014;23 Suppl 39:S120-32.

4. Kavanaugh A, Tomar R, Reveille J, Solomon DH, Homburger HA. Guidelines for clinical use of the antinuclear antibody test and tests for specific autoantibodies to nuclear antigens. American College of Pathologists. Arch Pathol Lab Med 2000;124:71-81.

5. Isenberg DA, Manson JJ, Ehrenstein MR, Rahman A. Fifty years of anti-ds DNA antibodies: are we approaching journey's end? Rheumatology 2007;46:1052-6.

6. Pan N, Amigues I, Lyman S, Duculan R, Aziz F, Crow MK, et al. A surge in anti-dsDNA titer predicts a severe lupus flare within six months. Lupus 2014;23:293-8.

7. Linnik MD, Hu JZ, Heilbrunn KR, Strand V, Hurley FL, Joh T. Relationship between anti-double-stranded DNA antibodies and exacerbation of renal disease in patients with systemic lupus erythematosus. Arthritis Rheum 2005;52:1129-37.

8. ter Borg EJ, Horst G, Hummel EJ, Limburg PC, Kallenberg CG. Measurement of increases in anti-double-stranded DNA antibody levels as a predictor of disease exacerbation in systemic lupus erythematosus. A long-term, prospective study. Arthritis Rheum 1990;33:634-43.

9. Cozzani E, Drosera M, Gasparini G, Parodi A. Serology of lupus erythematosus: correlation between immunopathological features and clinical aspects. Autoimmune Dis 2014;2014:321359.

10. Ho A, Barr SG, Magder LS, Petri M. A decrease in complement is associated with increased renal and hematologic activity in patients with systemic lupus erythematosus. Arthritis Rheum 2001;44:2350-7.

11. Giles BM, Boackle SA. Linking complement and anti-dsDNA antibodies in the pathogenesis of systemic lupus erythematosus. Immunol Res 2013;55:10-21.

12. Swaak AJ, Groenwold J, Bronsveld W. Predictive value of complement profiles and anti-dsDNA in systemic lupus erythematosus. Ann Rheum Dis 1986;45:359-66.

13. Hochberg MC. Updating the American College of Rheumatology revised criteria for the classification of systemic lupus erythematosus. Arthritis Rheum 1997;40:1725.

14. Tan EM, Cohen AS, Fries JF, Masi AT, McShane DJ, Rothfield NF, et al. The 1982 revised criteria for the classification of systemic lupus erythematosus. Arthritis Rheum 1982;25:1271-7.

15. Petri M, Orbai AM, Alarcón GS, Gordon C, Merrill JT, Fortin PR, et al. Derivation and validation of the Systemic Lupus International Collaborating Clinics classification criteria for systemic lupus erythematosus. Arthritis Rheum 2012;64:2677-86

16. Gladman DD, Ibañez D, Urowitz MB. Systemic lupus erythematosus disease activity index 2000. J Rheumatol 2002;29:288-91.

17. Man A, Shojania K, Phoon C, Pal J, de Badyn MH, Pi D, et al. An evaluation of autoimmune antibody testing patterns in a Canadian health region and an evaluation of a laboratory algorithm aimed at reducing unnecessary testing. Clin Rheumatol 2013;32:601-8.

18. Bonaguri C, Melegari A, Ballabio A, Parmeggiani M, Russo A, Battistelli L, et al. Italian multicentre study for application of a diagnostic algorithm in autoantibody testing for autoimmune rheumatic disease: conclusive results. Autoimmun Rev 2011;11:1-5.

Personal non-commercial use only. The Journal of Rheumatology Copyright (C) 2018. All rights reserved. 
19. Solomon DH, Kavanaugh AJ, Schur PH; American College of Rheumatology Ad Hoc Committee on Immunologic Testing Guidelines. Evidence-based guidelines for the use of immunologic tests: antinuclear antibody testing. Arthritis Rheum 2002;47:434-44.

20. Faria AC, Barcellos KS, Andrade LE. Longitudinal fluctuation of antibodies to extractable nuclear antigens in systemic lupus erythematosus. J Rheumatol 2005;32:1267-72.

21. Tench CM, Isenberg DA. The variation in anti-ENA characteristics between different ethnic populations with systemic lupus erythematosus over a 10-year period. Lupus 2000;9:374-6.

22. Praprotnik S, Bozic B, Kveder T, Rozman B. Fluctuation of anti-Ro/SS-A antibody levels in patients with systemic lupus erythematosus and Sjögren's syndrome: a prospective study. Clin Exp Rheumatol 1999;17:63-8.

23. Chow SL, Carter Thorne J, Bell MJ, Ferrari R, Bagheri Z, Boyd T, et al; Canadian Rheumatology Association Choosing Wisely Committee. Choosing wisely: the Canadian Rheumatology
Association's list of 5 items physicians and patients should question. J Rheumatol 2015;42:682-9.

24. Center for Medicaid \& Medicare Services. Clinical Laboratory Fee Schedule 16CLAB Revised for January 2016. [Internet. Accessed February 2, 2018.] Available from: www.cms.gov/Medicare/ Medicare-Fee-for-Service-Payment/ClinicalLabFeeSched/ Clinical-Laboratory-Fee-Schedule-Files.html

25. Agarwal S, Harper J, Kiely PD. Concentration of antibodies to extractable nuclear antigens and disease activity in systemic lupus erythematosus. Lupus 2009;18:407-12.

26. Lateef A, Petri M. Managing lupus patients during pregnancy. Best Pract Res Clin Rheumatol 2013;27:435-47.

27. Blomberg S, Ronnblom L, Wallgren AC, Nilsson B, Karlsson-Parra A. Anti-SSA/Ro antibody determination by enzyme-linked immunosorbent assay as a supplement to standard immunofluorescence in antinuclear antibody screening. Scand J Immunol 2000;51:612-7. 